\title{
Smooth Adaptive Internal Model Control Based on $U$ Model for Nonlinear Systems with Dynamic Uncertainties
}

\author{
Li Zhao, ${ }^{1}$ Jing Wang, ${ }^{1}$ and Weicun Zhang $^{2}$ \\ ${ }^{1}$ Engineering Research Institute, University of Science and Technology Beijing, Beijing 100083, China \\ ${ }^{2}$ School of Automation \& Electrical Engineering, University of Science and Technology Beijing, Beijing 100083, China \\ Correspondence should be addressed to Li Zhao; zhaoliae86@vip.163.com
}

Received 22 June 2016; Accepted 27 September 2016

Academic Editor: Tarek Ahmed-Ali

Copyright (c) 2016 Li Zhao et al. This is an open access article distributed under the Creative Commons Attribution License, which permits unrestricted use, distribution, and reproduction in any medium, provided the original work is properly cited.

\begin{abstract}
An improved smooth adaptive internal model control based on $U$ model control method is presented to simplify modeling structure and parameter identification for a class of uncertain dynamic systems with unknown model parameters and bounded external disturbances. Differing from traditional adaptive methods, the proposed controller can simplify the identification of time-varying parameters in presence of bounded external disturbances. Combining the small gain theorem and the virtual equivalent system theory, learning rate of smooth adaptive internal model controller has been analyzed and the closed-loop virtual equivalent system based on discrete $U$ model has been constructed as well. The convergence of this virtual equivalent system is proved, which further shows the convergence of the complex closed-loop discrete $U$ model system. Finally, simulation and experimental results on a typical nonlinear dynamic system verified the feasibility of the proposed algorithm. The proposed method is shown to have lighter identification burden and higher control accuracy than the traditional adaptive controller.
\end{abstract}

\section{Introduction}

During the past few decades, the rapid development of modern industry urgently needs the improvement of the production efficiency and quality. And the modern industrial control process is a class of nonlinear dynamic systems, which typically have characteristics of time-varying complexity and diversity [1]. Therefore, a nonlinear model with wide applicability and simple structure is essential to solve control system design problems caused by various uncertainties.

In order to improve the dynamic performance and control precision for industrial systems, it is important to establish an accurate dynamic model which can well represent its dynamic characteristics [2]. An accurate dynamics model is a precondition of dynamic performance analysis and precise control. The system dynamic performance can be calculated by mechanism modeling [3]. Meanwhile, the accurate parameters of the process system can be obtained via system identification methods. Therefore, modeling technology has become an important research content in the field of industrial system control.
However, the precise mechanism of modeling based on the energy conservation law and classical physics cannot resolve complex industrial process completely [4]. Traditional mechanism modeling of numerical calculation has difficulties to deal with a variety of information in complex industrialized environmental systems and to fully reflect the industrial process systems [5]. For systems with unknown parameters and bounded disturbances, the modern system modeling developed mathematical modeling and model validation.

A suit of considerable theoretical methods have been developed for nonlinear system modeling, such as the nonlinear FIR model, finite Volterra model [6], Hammerstein model [7], and Wiener model [8]. In 2002, professor Zhu first proposed the theory of $U$ model [9], which can be seen as a special deformation structure of nonlinear autoregressive moving average with exogenous inputs (NARMAX) model, with characteristics of simple structure and wide range [10]. In 2005 Shafiq and Haseebuddin proposed $U$ model-based internal model control for nonlinear dynamic plants, and the system achieved good robustness for both 
linear systems and nonlinear systems [11]. The $U$ model control system was expanded from single input single output (SISO) nonlinear system to multi-input multioutput (MIMO) nonlinear system based on MIMO $U$ model control strategy proposed by Azhar in 2008. But the MIMO $U$ model system needs a lot of data identification calculations, which costs huge time and mechanical energy [12]. Up to now model control algorithms have obtained numerous achievements; however, the convergence study of the closed-loop system is still an open problem for the $U$ model theoretical research [13].

How to combine the modeling theory and the intelligent control theory for industrial process control is a practical problem, and it is a theoretical problem to analyze the convergence of industrial control system [14]. A smooth adaptive internal model control method based on $U$ model is proposed. For time-varying parameters of nonlinear dynamic system, the proposed control method based on $U$ model simplifies the structure of the nonlinear system. By establishing parameter error of the discrete $U$ model system and using the small gain theorem, the learning rate's stability is analyzed. Meanwhile, based on the closed-loop virtual equivalent system theory [15], the convergence proof of the $U$ model closed-loop system is completed. Besides, the simulation and experimental results show the effectiveness of the proposed algorithm.

The rest of the paper is organized as follows. In Section 2, the $U$ model structure is presented. In Section 3, a smooth adaptive internal model control system is proposed for $U$ model system, including the controller design and control input solutions. The details of convergence proof for the closed-loop $U$ model system are described in Section 4 . Finally, the effectiveness of the proposed control algorithm is verified by the simulation and experimental results in Section 5 and a brief conclusion is given in Section 6 .

\section{Mathematical Description of $U$ Model}

The common nonlinear dynamic system is described as a form of NARMAX model as follows:

$$
\begin{aligned}
& y(t)=f(y(t-1), \ldots, y(t-n), u(t-1), \ldots, u(t-n), \\
& \quad e(t), \ldots, e(t-n)),
\end{aligned}
$$

where $y(t)$ and $u(t)$ denote the discrete time system output and input at time $t$, respectively. $e(t)$ is the error signal caused by the system volume measured noise, model error, and uncertain dynamic factors. $n$ stands for the maximum delay of the whole system. $f(\cdot)$ denotes the unknown nonlinear smoothing function with any known delay.

Assumption 1. System inputs and outputs are measureable, and the output noise is bounded, but the function $f(\cdot)$ is unknown.

Assumption 2. The highest order of the system input is known.
The control goal is to keep the system output signal tracking the reference signal under initial condition via control input design.

By extending the nonlinear function $f(\cdot)$, the control input $u(t-1)$ is expressed as the power series expansion at time $t-1$. Meanwhile, the other signals are integrated into time-varying parameters of the system input, and expression (1) is reconstructed as follows:

$$
y(t)=\sum_{j=0}^{L} \alpha_{j}(t) u^{j}(t-1)+e(t),
$$

where $L$ denotes the degree of the control input $u(t-1)$. The time-varying parameter $\alpha_{j}(t)$ is the nonlinear function including the past time output $y(t-1), \ldots, y(t-n)$, the past time input $u(t-2), \ldots, u(t-n)$, and error signal $e(t-$ $1), \ldots, e(t-n)$ which can be described as

$$
\begin{aligned}
& \alpha_{j}(t)=\alpha_{j}(y(t-1), \ldots, y(t-n), u(t-2), \ldots, u(t-n), \\
& \quad e(t-1), \ldots, e(t-n)) .
\end{aligned}
$$

In order to consult the design of linear control system, a new variable $U(t)$ is introduced in the nonlinear model as follows:

$$
\begin{aligned}
y(t) & =U(t-1), \\
U(t-1) & =\sum_{j=0}^{L} \alpha_{j}(t) u^{j}(t-1)+e(t) .
\end{aligned}
$$

It is worth to notice that (5) is denoted as $U$ model or the time-varying polynomial function model [9].

In order to identify the dynamic parameters in the $U$ model, the weighted recursive least square (RLS) method is used. Since the "data saturation" may occur when the data is growing, the updating function of the parameter estimates will be much weaker, so a forgetting parameter $\lambda$ is introduced [16]:

$$
\begin{aligned}
K_{k} & =\frac{P_{k} \Psi_{k}^{T}}{\lambda+\Psi_{k} P_{k} \Psi_{k}^{T}}, \\
\varepsilon_{k} & =Y_{k}-\Psi_{k} \widehat{\Theta}_{k}, \\
\widehat{\Theta}_{k+1} & =\widehat{\Theta}_{k}+K_{k} \varepsilon_{k}, \\
P_{k+1} & =P_{k}-K_{k} \Psi_{k} P_{k},
\end{aligned}
$$

where $K_{k} \in R^{L+1}$ denotes the system iterative weighted coefficient. $\varepsilon_{k}$ denotes error, including the output error and identification error of the system. According to formula (5), $\widehat{\Theta}_{k} \in R^{L+1}$ denotes identification vector of the system parameters $\left[\begin{array}{llll}\alpha_{0} & \alpha_{1} & \cdots & \alpha_{L}\end{array}\right] . \Psi_{k} \in R^{L+1}$ denotes the system input vector defined by $\left[\begin{array}{llll}1 & u_{1} & \cdots & u_{L}\end{array}\right]$ at time $t-1 . P_{k} \in$ $R^{(L+1) \times(L+1)}$ denotes covariance matrix.

The energy performance index $J$ can be derived:

$$
J=\sum_{k=1}^{L} \lambda^{L-k}\left[Y_{k}-\Psi_{k} \widehat{\Theta}_{k}\right]^{2} .
$$




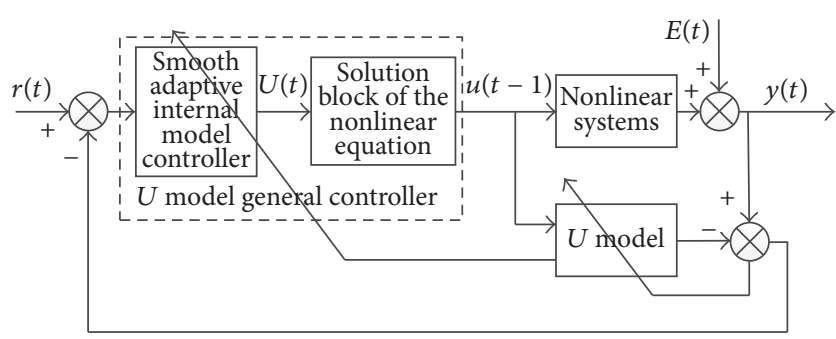

FIGURE 1: The smooth adaptive internal model controller based on $U$ model.

It should be noticed that when the forgetting parameter $\lambda(0<\lambda \leq 1)$ equals 1 , formula (6) will be simplified to a weighted recursive least square method.

The online identification results of $U$ model are obtained:

$$
\begin{aligned}
y(t) & =U(t-1), \\
U(t-1) & =\sum_{j=0}^{L} \widehat{\alpha}_{j}(t) u^{j}(t-1) .
\end{aligned}
$$

$U$ model is a special structure form of NARMAX model, which not only has a simple structure but also can be widely applied to many nonlinear systems. Furthermore, the design of the $U$ model control system can refer to the design of linear control system for its special structure.

\section{Design of Smooth Adaptive Internal Model Controller Based on $U$ Model}

The nonlinear controller designed for the $U$ model takes advantage of internal model controller with simple calculation and good tracking performance. Because the $U$ model controller contains a pseudo input signal, the solution block is used to solve the real control input from a nonlinear polynomial function. The smooth adaptive internal model controller and the solution block of nonlinear equation constitute the $U$ model general controller. The design of the proposed control system is shown in Figure 1 [17].

Due to the pseudo input signal, the internal controller can be used in the controller, which greatly simplifies the complexity of the controller as follows:

$$
U(t-1)=r(t)-y(t)+y_{m}(t),
$$

where $r(t)$ denotes the reference target signal; $y(t)$ and $y_{m}(t)$ denote the output of the system and the output of the model. By solving the nonlinear equation, the real control input can be obtained.

With the Newton iteration method, the nonlinear equation of the pseudo input signal of the $U$ model is calculated as follows:

$$
\begin{aligned}
& u_{k+1}(t-1)=u_{k}(t-1) \\
& \quad-\left.\frac{\left[\sum_{j=0}^{M} \alpha_{j}(t) u_{k}^{j}(t-1)\right]-U(t-1)}{d\left[\sum_{j=0}^{M} \alpha_{j}(t) u^{j}(t-1)\right] / d u(t-1)}\right|_{u^{j}(t-1)=u_{k}^{j}(t-1)} .
\end{aligned}
$$

$k$ is the number of iterations.
Considering the impact from the disturbance, model mismatch, and the measurement noise, the disturbance $e(t)$ is estimated as follows:

$$
\widehat{e}(t)=\left[\sum_{j=0}^{M} \widehat{\alpha}_{j}(t) u_{k}^{j}(t-1)\right]-y(t) .
$$

$\widehat{\alpha}(t)$ denotes the estimated value of the system parameter $\alpha(t)$. By substituting $\widehat{\alpha}(t)$ into (10), we get

$$
\begin{aligned}
& u_{k+1}(t-1)=u_{k}(t-1) \\
& \quad-\left.\frac{\left[\sum_{j=0}^{M} \widehat{\alpha}_{j}(t) u_{k}^{j}(t-1)\right]-U(t-1)}{d\left[\sum_{j=0}^{M} \widehat{\alpha}_{j}(t) u_{k}^{j}(t-1)\right] / d u_{k}(t-1)}\right|_{u^{j}(t-1)=u_{k}^{j}(t-1)} .
\end{aligned}
$$

In order to optimize the Newton iteration solving equations, to reduce the root impact caused by the sudden system change, and to protect the application machine, smoothing factor $\gamma$ is introduced in the adaptive internal controller:

$$
\begin{aligned}
& u_{k+1}(t-1)=u_{k}(t-1) \\
& \quad-\left.\gamma \frac{\left[\sum_{j=0}^{M} \widehat{\alpha}_{j}(t) u_{k}^{j}(t-1)\right]-U(t-1)}{d\left[\sum_{j=0}^{M} \widehat{\alpha}_{j}(t) u_{k}^{j}(t-1)\right] / d u_{k}(t-1)}\right|_{u^{j}(t)=u_{k}^{j}(t)},
\end{aligned}
$$

where $\gamma>0$.

As $\gamma$ decreases, system convergence speed becomes slow, so the iterative algorithm can adapt to the slow changed system. If $\gamma$ is increased, the convergence speed of the system is accelerated, so the iterative algorithm can adapt to the system with fast response.

Meanwhile, in order to avoid the iterative algorithm breakdown when denominator approximates to zero, the range of denominator is redefined as shown in Figure 2. The specific calculation process is that we set $k=0$ and $l=0$, which denote the main initial cycle time and collateral initial cycle time. Calculate the identified $U$ model output $\Phi(t)$ and its derivative $\dot{\Phi}(t)$. Then the initial values are that $D_{1}=4$, $D_{2}=0, D_{3}=3$, and $D_{4}$ is a constant that approximates to zero and starts the calculation. If the absolute value of the derivative $|\dot{\Phi}(t)|$ is not less than $D_{4}$, the iteration goes on. If the absolute value of the derivative is less than $D_{4}$, the iterative value will be modified. The calculating process is that if $\Phi(t)$ is less than $D_{2}, \Phi(t)$ will be the last iteration value and the calculation will be recalculated from the beginning. If $\Phi(t)$ is larger than $D_{2}$, the program will continue to judge collateral cycle time $l$ and $D_{3}$. If $l$ is less than $D_{3}, l=l+1$. If $l$ is larger than $D_{3}$, the calculation is complete and the control input $u(t-1)$ is returned.

In order to guarantee the stability of the smooth adaptive internal model control algorithm based on $U$ model, the range of learning rate is given. First, the parameter identification error is calculated.

According to (2), the vector form of $U$ model is expressed:

$$
y(t)=\Theta(t) \Psi(t)+e(t),
$$

where $\Theta(t)=\left[\alpha_{0}(t), \alpha_{1}(t), \ldots, \alpha_{M}(t)\right], \Psi(t)=[1, u(t-$ $\left.1), u^{2}(t-1), \ldots, u^{M}(t-1)\right]^{T}$, and the error $e(t)$ comes 


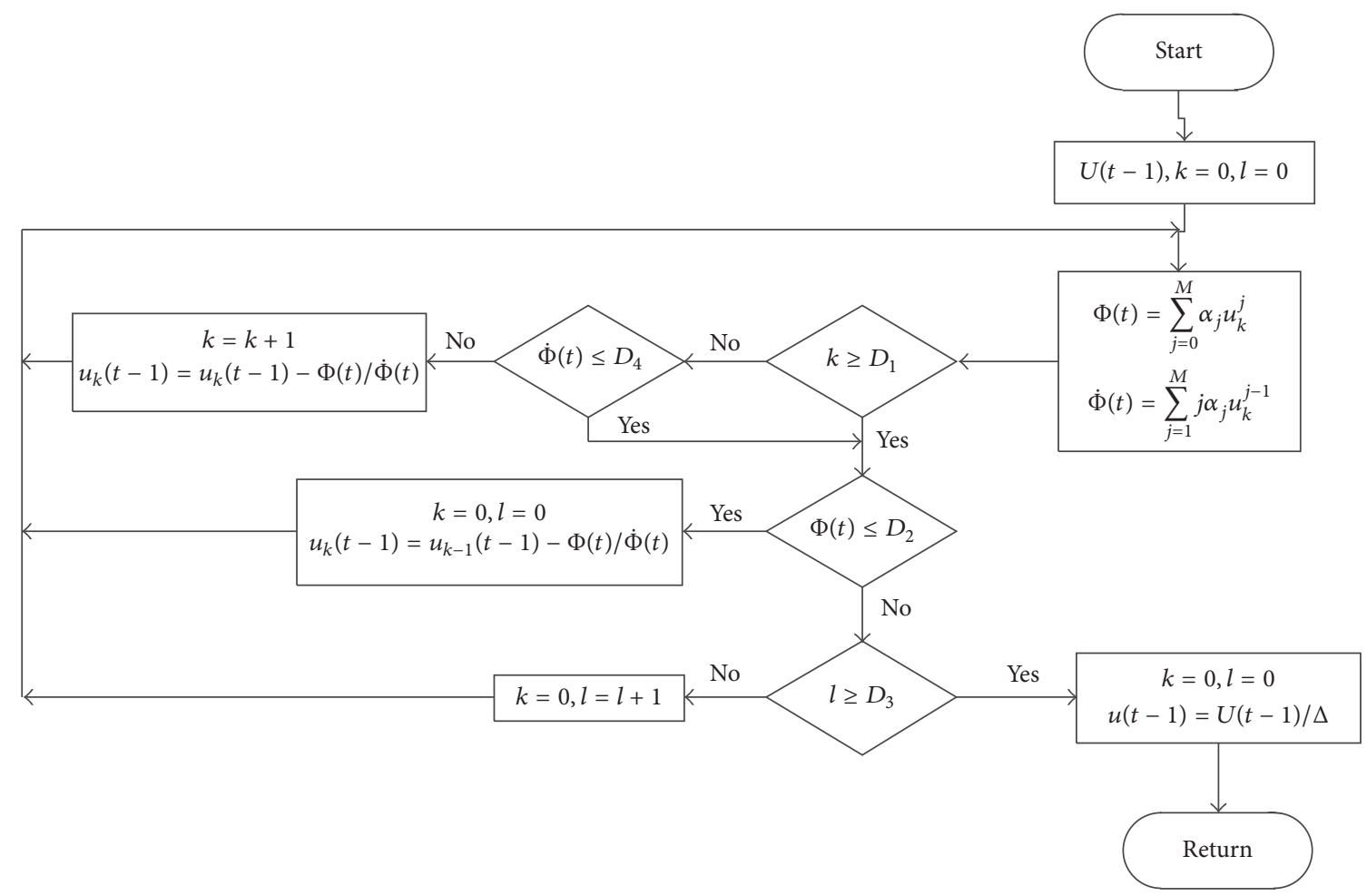

FIGURE 2: The smooth adaptive internal model controller process.

from the model mismatch and the measurement noise. Thus, the system identification problem can be transformed into the estimation of the parameters $\Theta(t)$ of $U$ model. The identification system of $U$ model is expressed as

$$
y_{m}(t)=\widehat{\Theta}(t) \Psi(t) .
$$

The identification parameter $\widehat{\Theta}(t)$ estimates the system parameter $\Theta(t)$ at time $t$. If the initial identification parameter is $\widehat{\Theta}_{0}(t)$, the identification parameter updated by the weighted recursive least square method with forgetting parameter will be as follows:

$$
\widehat{\Theta}_{k+1}(t)=\widehat{\Theta}_{k}(t)+K_{t} \varepsilon(t),
$$

among which,

$$
\begin{aligned}
\varepsilon(t) & =y(t)-y_{m}(t)+e(t) \\
& =\Theta(t) \Psi(t)-\widehat{\Theta}(t) \Psi(t)+e(t),
\end{aligned}
$$

where $e(t)$ is the total disturbance of the system, including the measurement noise and external load disturbance.

The estimated parameter error vector is $\widetilde{\Theta}_{k}(t)=\Theta(t)-$ $\widehat{\Theta}_{k}(t)$ and a priori error $e_{a}(t)$ and a posterior error $e_{b}(t)$ can be expressed as

$$
\begin{aligned}
e_{a}(t) & =\widetilde{\Theta}_{k}(t) \Psi(t)=\Theta(t) \Psi(t)-\widehat{\Theta}_{k}(t) \Psi(t) \\
& =\Theta(t) \Psi(t)-y_{m}(t), \\
e_{b}(t) & =\widetilde{\Theta}_{k+1}(t) \Psi(t)=\Theta(t) \Psi(t)-\widehat{\Theta}_{k+1}(t) \Psi(t) \\
& =\Theta(t) \Psi(t)-\left[\widehat{\Theta}_{k}(t)+K_{t} \varepsilon(t)\right] \Psi(t) .
\end{aligned}
$$

The recursive expression of the estimated parameter error vector is given as follows:

$$
\begin{aligned}
\widetilde{\Theta}_{k+1}(t) & =\Theta(t)-\widehat{\Theta}_{k+1}(t) \\
& =\Theta(t)-\left[\widehat{\Theta}_{k}(t)+K_{t} \varepsilon(t)\right] \\
& =\Theta(t)-\left[\Theta(t)-\widetilde{\Theta}_{k}(t)+K_{t} \varepsilon(t)\right] \\
& =\widetilde{\Theta}_{k}(t)-K_{t} \varepsilon(t) .
\end{aligned}
$$

According to the small gain theory, when the estimation error energy divided by disturbance energy is less than 1 , the controller estimation error energy is less than the disturbance energy, no matter which disturbance it is:

$$
\frac{E_{e}}{E_{d}} \leq 1
$$

$E_{e}$ is the estimate error energy and $E_{d}$ is the total disturbance energy of the system.

From (17) and (18), the total disturbance $e(t)$ of the system is

$$
e(t)=\varepsilon(t)-e_{a}(t) .
$$

The energy norm of the estimate parameters error equation is obtained:

$$
\begin{aligned}
\left\|\widetilde{\Theta}_{k+1}(t)\right\|^{2}= & \left\|\widetilde{\Theta}_{k}(t)\right\|^{2}-2 \widetilde{\Theta}_{k}(t) K_{t} \varepsilon(t)+\left[K_{t} \varepsilon(t)\right]^{2} \\
= & \left\|\widetilde{\Theta}_{k}(t)\right\|^{2}-2 \widetilde{\Theta}_{k}(t) K_{t} \varepsilon(t) \\
& +K_{t}^{2}\left[e(t)+e_{a}(t)\right]^{2} .
\end{aligned}
$$


To make the efficiency of identification calculation adjustable, a new learning rate variable $\mu(t)$ is introduced in (16). And the updating equation of the identification parameter will be as follows:

$$
\widehat{\Theta}_{k+1}(t)=\widehat{\Theta}_{k}(t)+\mu(t) K_{t} \varepsilon(t) .
$$

Substituted it into (23):

$$
\begin{aligned}
\left\|\widetilde{\Theta}_{k+1}(t)\right\|^{2}= & \left\|\widetilde{\Theta}_{k}(t)\right\|^{2}-2 \widetilde{\Theta}_{k}(t) \mu(t) \varepsilon(t) \Psi(t-1) \\
& +\mu^{2}(t)\left[e(t)+e_{a}(t)\right]^{2}\|\Psi(t-1)\|^{2} .
\end{aligned}
$$

According to (18) (25),

$$
\begin{aligned}
& e_{a}(t)=\widetilde{\Theta}_{k}(t) \Psi(t-1) \\
& \left\|\widetilde{\Theta}_{k+1}(t)\right\|^{2}+2 \mu(t) e_{a}^{2}(t)-\mu^{2}(t) e_{a}^{2}(t)\|\Psi(t-1)\|^{2} \\
& =\left\|\widetilde{\Theta}_{k}(t)\right\|^{2}+2 \mu^{2}(t) e_{a}(t) e(t)\|\Psi(t-1)\|^{2} \\
& \quad-2 \mu(t) e_{a}(t) e(t)+\mu^{2}(t) e^{2}(t)\|\Psi(t-1)\|^{2}
\end{aligned}
$$

The input energy norm is as follows:

$$
\omega(t)=\|\Psi(t-1)\|^{2} .
$$

Thus, we can have

$$
\begin{gathered}
\left\|\widetilde{\Theta}_{k+1}(t)\right\|^{2}+2 \mu(t) e_{a}^{2}(t)-\mu^{2}(t) e_{a}^{2}(t) \omega(t) \\
=\left\|\widetilde{\Theta}_{k}(t)\right\|^{2}+2 \mu^{2}(t) e_{a}(t) e(t) \omega(t) \\
-2 \mu(t) e_{a}(t) e(t)+\mu^{2}(t) e^{2}(t) \omega(t) .
\end{gathered}
$$

When $\mu(t)=1 / \omega(t),(29)$ can be rewritten as

$$
\begin{aligned}
& \left\|\widetilde{\Theta}_{k+1}(t)\right\|^{2}+2 \mu(t) e_{a}^{2}(t)-\mu(t) e_{a}^{2}(t) \\
& =\left\|\widetilde{\Theta}_{k}(t)\right\|^{2}+2 \mu(t) e_{a}(t) e(t)-2 \mu(t) e_{a}(t) e(t) \\
& \quad+\mu(t) e^{2}(t) .
\end{aligned}
$$

By simplifying (30), we have

$$
\left\|\widetilde{\Theta}_{k+1}(t)\right\|^{2}+\mu(t) e_{a}^{2}(t)=\left\|\widetilde{\Theta}_{k}(t)\right\|^{2}+\mu(t) e^{2}(t) .
$$

If the energy condition always satisfies $E_{e}=E_{d}$, the range of learning rate is calculated as follows:

$$
\frac{\left\|\widetilde{\Theta}_{k+1}(t)\right\|^{2}+\mu(t) e_{a}^{2}(t)}{\left\|\widetilde{\Theta}_{k}(t)\right\|^{2}+\mu(t) e^{2}(t)} \begin{cases}\leq 1 & 0<\mu(t)<\frac{1}{\omega(t)} \\ =1 & \mu(t)=\frac{1}{\omega(t)} \\ \geq 1 & \mu(t)>\frac{1}{\omega(t)} .\end{cases}
$$

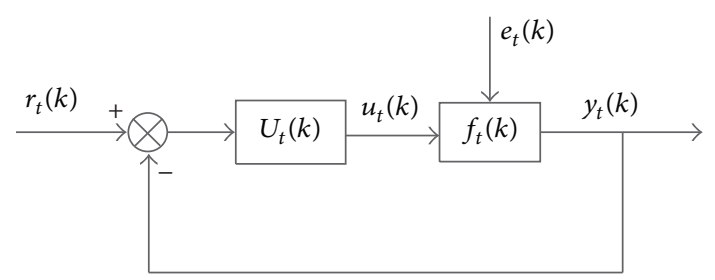

FIGURE 3: The control system based on $U$ model.

If the learning rate is selected to satisfy $\mu(t) \leq 1 / \omega(t)$, from the energy point of view, the signal $\left\{\widetilde{\Theta}_{k+1}(t), \sqrt{\mu(t)} e_{a}(t)\right\}$ to the signal $\left\{\widetilde{\Theta}_{k}(t), \sqrt{\mu(t)} e(t)\right\}$ is convergence mapping, which can improve the robustness of the identification parameter. At the same time, the energy condition also shows that no matter how much the disturbance $e(t)$ is, and no matter how much the difference between the identification parameters $\widehat{\Theta}(t)$ and the real parameters $\Theta(t)$, the energy $\left\|\widetilde{\Theta}_{k+1}(t)\right\|^{2}+\mu(t) e_{a}{ }^{2}(t)$ will be always less than the energy $\left\|\widetilde{\Theta}_{k}(t)\right\|^{2}+\mu(t) e^{2}(t)$.

From the above analyses, we can get the range of learning rate of identification parameters, so as to ensure the stability range of learning rate for the smooth adaptive internal model controller based on $U$ model.

With the smooth adaptive internal model controller based on $U$ model, the modeling theory and the intelligent nonlinear controller for practical industrial process problems are efficiently combined. For nonlinear systems with unknown model parameters and bounded external disturbances, the proposed method which has a tunable learning rate and simplifies identification structure is different from the traditional adaptive controller.

\section{The Convergence Proof}

For convergence of the improved smooth adaptive internal model based on $U$ model control system, Section 3 presents the stability range of the controller, which is the sufficient condition for Section 4 . In Section 4 , by the theory of virtual equivalent system, the improved smooth adaptive internal model based on $U$ model system is reconstructed into a virtual equivalent system and the stability and convergence proof are accomplished.

Because the controlled system is nonlinear dynamic with large uncertainties, it is difficult to finish the convergence proof for $U$ model closed-loop control system directly. Thus, by the theory of virtual equivalent system, the closed-loop control system is discretized at the time $t$ shown in Figure 3.

In Figure $3, f_{t}(k)$ is the practical controlled object at time t. $U_{t}(k)$ is the $U$ model general controller. $e_{t}(k)$ is the white noise.

First, the virtual equivalent system is constructed as shown in Figure 4, where $e_{c t}(k)=y_{t}(k)-\Theta_{t}(k) \Psi_{t}(k)-e_{t}(k)$ is the parameter estimation error at time $t . f_{m t}(k)$ is the identification model at time $t$.

Then the construction of the "slow switching" virtual equivalent system is shown in Figure 5. 


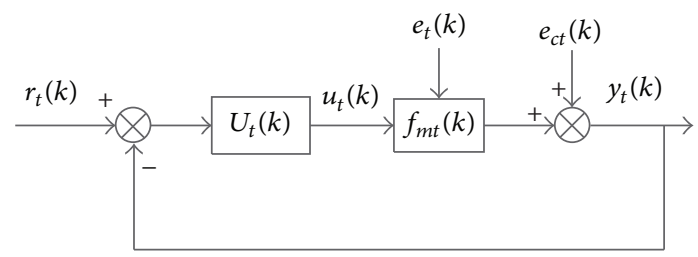

FIGURE 4: The virtual equivalent system I.

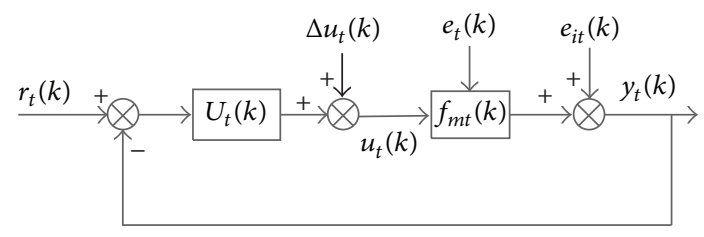

FIGURE 5: The virtual equivalent system II.

In Figure 5,

$$
\begin{aligned}
e_{i t}(k)= & y_{t}(k)-\widehat{\Theta}_{t}\left(l_{k}\right) \Psi_{t}(k)-e_{t}(k) \\
= & y_{t}(k)-\widehat{\Theta}_{t}(k) \Psi_{t}(k)-e_{t}(k) \\
& +\left[\widehat{\Theta}_{t}(k)-\widehat{\Theta}_{t}\left(l_{k}\right)\right] \Psi_{t}(k) \\
= & e_{c t}(k)+\left[\widehat{\Theta}_{t}(k)-\widehat{\Theta}_{t}\left(l_{k}\right)\right] \Psi_{t}(k), \\
\Delta u_{t}(k)= & \Theta_{c t}(k) \Psi_{c t}(k)-\Theta_{c t}\left(l_{k}\right) \Psi_{c t}(k)
\end{aligned}
$$

$\Theta_{c t}(k)=f\left(\widehat{\Theta}_{t}(k)\right)$ is the parameter vector for $U$ model general controller $U_{t}(k)$ at time $t . \Psi_{c t}(k)$ is the control input regression vector.

The convergence of closed-loop $U$ model control system is that the output $y_{t}(k)$ of the practical controlled object can track the reference target $r_{t}(k)$.

Theorem 3. For uncertain nonlinear dynamic $U$ model system, the system is stable and locally convergent if it can satisfy conditions as follows:

(1) $\left\|\widehat{\Theta}_{t}(k)\right\| \leq M<\infty ;\left\|\widehat{\Theta}_{t}(k)-\widehat{\Theta}_{t}(k-m)\right\| \rightarrow 0$, $m$ is $a$ bounded value.

(2) $\sum_{k=1}^{n}\left\|e_{c t}(k)\right\|^{2}=o\left(\delta+\sum_{k=1}^{n}\left\|\Psi_{t}(k)\right\|^{2}\right), 0<\delta<\infty$.

(3) The U model controller is well defined such that $\sum\{U, f\}$ constitutes a stable closed-loop system and can track the bounded reference target signal with the control strategy.

(4) $f\left(\widehat{\Theta}_{t}(k)\right)$ is a continuous function for $\widehat{\Theta}_{t}(k)$.

Proof. (i) In order to validate the system's stability, the virtual equivalent system is decomposed into three subsystems, which are shown from Figures 6 to 8 .

According to (20), it satisfies the theorem conditions (1). Meanwhile, according to the stability range of learning rate value in Section 3, it can satisfy the theorem conditions (3).

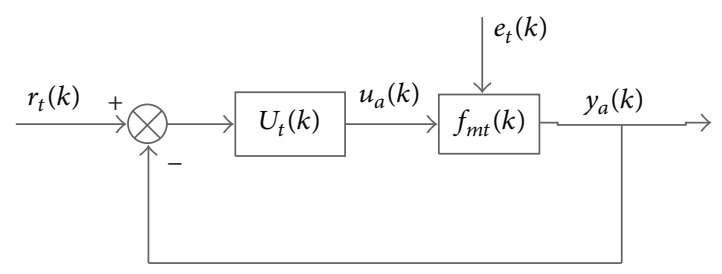

FIgURE 6: The decomposition subsystem I.

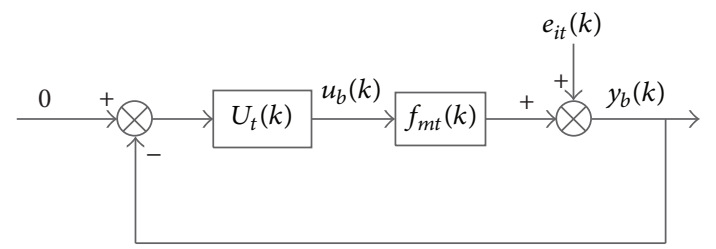

FIGURE 7: The decomposition subsystem II.

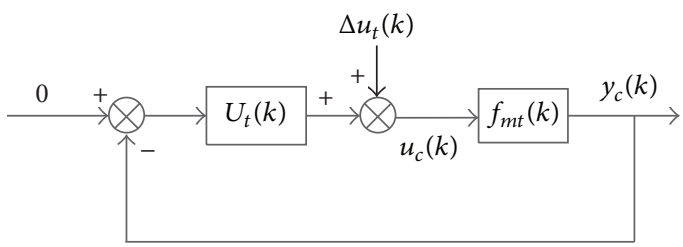

FIgURE 8: The decomposition subsystem III.

Theorem conditions (1) and (3) can guarantee the system to be a stable "slow switching" control system:

$$
\begin{array}{r}
\frac{1}{n} \sum_{k=1}^{n}\left(\left\|y_{a}(k)\right\|^{2}+\left\|u_{a}(k)\right\|^{2}\right)<\infty, \\
\frac{1}{n} \sum_{k=1}^{n}\left\|y_{a}(k)-r_{t}(k)\right\|^{2}<\infty .
\end{array}
$$

There are two compensation signals which can be obtained by theorem conditions (1) and (3):

$$
\begin{gathered}
\frac{1}{n} \sum_{k=1}^{n}\left\|e_{i t}(k)\right\|^{2}=o\left(\frac{1}{n} \sum_{k=1}^{n}\left\|\Psi_{t}(k)\right\|^{2}\right), \\
\frac{1}{n} \sum_{k=1}^{n}\left\|\Delta u_{t}(k)\right\|^{2}=o\left(\frac{1}{n} \sum_{k=1}^{n}\left\|\Psi_{t}(k)\right\|^{2}\right) .
\end{gathered}
$$

Because the subsystem II and the subsystem III are both stable systems, so the following equations can be derived:

$$
\begin{aligned}
& \frac{1}{n} \sum_{k=1}^{n}\left\|y_{b}(k)\right\|^{2}=o\left(\frac{1}{n} \sum_{k=1}^{n}\left\|\Psi_{t}(k)\right\|^{2}\right), \\
& \frac{1}{n} \sum_{k=1}^{n}\left\|u_{b}(k)\right\|^{2}=o\left(\frac{1}{n} \sum_{k=1}^{n}\left\|\Psi_{t}(k)\right\|^{2}\right), \\
& \frac{1}{n} \sum_{k=1}^{n}\left\|y_{c}(k)\right\|^{2}=o\left(\frac{1}{n} \sum_{k=1}^{n}\left\|\Psi_{t}(k)\right\|^{2}\right),
\end{aligned}
$$




$$
\frac{1}{n} \sum_{k=1}^{n}\left\|u_{c}(k)\right\|^{2}=o\left(\frac{1}{n} \sum_{k=1}^{n}\left\|\Psi_{t}(k)\right\|^{2}\right)
$$

For the stability proof, consider

$$
\begin{aligned}
& y_{t}(k)=y_{a}(k)+y_{b}(k)+y_{c}(k), \\
& u_{t}(k)=u_{a}(k)+u_{b}(k)+u_{c}(k) .
\end{aligned}
$$

Considering $\Psi_{t}(k), \Psi_{t 1}(k)$ can be constructed from subsystem I, $\Psi_{t 2}(k)$ can be constructed from the subsystem II, and $\Psi_{t 3}(k)$ can be constructed from subsystem III.

$$
\Psi_{t}(k)=\Psi_{t 1}(k)+\Psi_{t 2}(k)+\Psi_{t 3}(k) .
$$

From (38) to (41),

$$
\begin{aligned}
& \frac{1}{n} \sum_{k=1}^{n}\left\|\Psi_{t 2}(k)\right\|^{2}=o\left(\frac{1}{n} \sum_{k=1}^{n}\left\|\Psi_{t}(k)\right\|^{2}\right), \\
& \frac{1}{n} \sum_{k=1}^{n}\left\|\Psi_{t 3}(k)\right\|^{2}=o\left(\frac{1}{n} \sum_{k=1}^{n}\left\|\Psi_{t}(k)\right\|^{2}\right) .
\end{aligned}
$$

Rewrite (34):

$$
\frac{1}{n} \sum_{k=1}^{n}\left\|\Psi_{t 1}(k)\right\|^{2}<\infty
$$

This virtual equivalent system can get the following equation:

$$
\frac{1}{n} \sum_{k=1}^{n}\left\|\Psi_{t}(k)\right\|^{2}<\infty .
$$

Thus, the stability of the control system is proved.

(ii) In order to prove the convergence, considering (39) to (42) and using (48), the following equation can be derived [18]:

$$
\begin{aligned}
& \frac{1}{n} \sum_{k=1}^{n}\left\|y_{b}(k)\right\|^{2}=o(1), \\
& \frac{1}{n} \sum_{k=1}^{n}\left\|u_{b}(k)\right\|^{2}=o(1), \\
& \frac{1}{n} \sum_{k=1}^{n}\left\|y_{c}(k)\right\|^{2}=o(1), \\
& \frac{1}{n} \sum_{k=1}^{n}\left\|u_{c}(k)\right\|^{2}=o(1) .
\end{aligned}
$$

And then the equation is obtained as follows:

$$
\begin{aligned}
& \frac{1}{n} \sum_{k=1}^{n}\left\|y_{t}(k)-r_{t}(k)\right\|^{2} \\
& \quad=\frac{1}{n} \sum_{k=1}^{n}\left\|y_{a}(k)-r_{t}(k)+y_{b}(k)+y_{c}(k)\right\|^{2} \longrightarrow \\
& \frac{1}{n} \sum_{k=1}^{n}\left\|y_{a}(k)-r_{t}(k)\right\|^{2} .
\end{aligned}
$$

The local convergence of the control system is proved.

\section{Simulation and Experimental Results}

In order to verify the effectiveness of the proposed control algorithm, a typical nonlinear system and an experimental example are selected. In the simulation the traditional adaptive control algorithm has been compared as well.

5.1. Simulation. The industrial electric heating system is a continuous system with dead time and its characteristic parameters changed with temperature, so it is difficult to get a precise mathematical model expression. Considering the complex industrial slab-heating temperature control system [19], the system model is

$$
\begin{aligned}
y(t)= & 0.6762 y(t-1) e(t-1)-0.5288 y^{2}(t-2) \\
& +0.0673 y(t-2) u(t-2) \\
& +0.6513 y^{2}(t-1) u(t-2) e(t-1) \\
& +0.9939 y^{2}(t-2) e(t-1) \\
& +0.3388 u^{2}(t-2) e(t-1)+0.7578 u(t-1) \\
& -0.2203 u(t-1) y^{2}(t-1) e(t-1) \\
& +0.6067 u(t-1) y(t-1) u^{2}(t-2) \\
& +0.2070 u(t-1) y(t-1) u^{2}(t-2) e(t-1) \\
& +0.0105 u^{2}(t-1) y(t-1) e(t-1) \\
& +0.9072 u^{3}(t-1) u(t-2) e(t-1) e(t-2) \\
& +e(t),
\end{aligned}
$$

where $e(t)$ is the Gauss noise, which represents the unknown disturbance and random noise. $y(t)$ is the temperature of the controlled object, and $u(t)$ is the control input that represents the controllable heating power. Rewrite (53) in the $U$ model structure as follows:

$$
\begin{aligned}
U(t)= & \alpha_{0}(t)+\alpha_{1}(t) u(t-1)+\alpha_{2}(t) u^{2}(t-1) \\
& +\alpha_{3}(t) u^{3}(t-1), \\
y(t)= & U(t) .
\end{aligned}
$$

It should be noticed that

$$
\begin{aligned}
\alpha_{0}(t)= & 0.6762 y(t-1) e(t-1)-0.5288 y^{2}(t-2) \\
& +0.0673 y(t-2) u(t-2) \\
& +0.6513 y^{2}(t-1) u(t-2) e(t-1) \\
& +0.9939 y^{2}(t-2) e(t-1) \\
& +0.3388 u^{2}(t-2) e(t-1)+e(t), \\
\alpha_{1}(t)= & 0.7578-0.2203 y^{2}(t-1) e(t-1) \\
& +0.6067 y(t-1) u^{2}(t-2) \\
& +0.2070 y(t-1) u^{2}(t-2) e(t-1),
\end{aligned}
$$


TABLE 1: The initial values of simulation controller.

\begin{tabular}{lccc}
\hline \multicolumn{2}{c}{ Smooth adaptive internal model controller based on $U$ model } & The traditional adaptive controller \\
\hline The number of identification parameters & 4 & The number of identification parameters & 12 \\
Initial values & $(0.8,0.8,0.8,0.8)$ & Initial values & $(0.8, \ldots, 0.8)$ \\
Forgetting parameter & $\lambda=0.95$ & Forgetting parameter & $\lambda=0.95$ \\
$P(0)$ & $I \times 10^{6}$ & $P(0)$ & $I \times 10^{6}$ \\
Learning rate & $\mu=0.35$ & - & - \\
Smoothing factors & $\gamma=1.2$ & - \\
\hline
\end{tabular}

$$
\begin{aligned}
& \alpha_{2}(t)=0.0105 y(t-1) e(t-1)_{2}, \\
& \alpha_{3}(t)=0.9072 u(t-2) e(t-1) e(t-2), \\
& \widehat{\Theta}(t)=\left[\widehat{\alpha}_{0}(t), \widehat{\alpha}_{1}(t), \widehat{\alpha}_{2}(t), \widehat{\alpha}_{3}(t)\right]^{T}, \\
& \widehat{\Psi}(t)=\left[1, u(t-1), u^{2}(t-1), u^{3}(t-1)\right] .
\end{aligned}
$$

According to the initial parameters of Table 1 [20], the reference trajectory $r(t)$ is a square wave signal, and the control target is to make the output temperature $y(t)$ tracking the reference trajectory. Simulation results are shown in Figure 9.

Figure 9 is the simulation results of the heating furnace temperature control system. (a) denotes the system output of the $U$ model smooth adaptive internal model controller when (b) is the system output of the traditional adaptive controller. Compared with the traditional adaptive controller, $U$ model smooth adaptive internal model controller has a simple structure, which can track the reference trajectory more accurately. (c) and (d) are the temperature difference, which shows that the $U$ model smooth adaptive internal model controller can keep the temperature in a low fluctuation. However, at the rising edge or falling edge, both $U$ model smooth adaptive internal model controller and traditional adaptive controller have a wide range of vibration.

For the industrial heating system, the identification of parameters of $U$ model structure is reduced to four, which greatly simplifies the structure complexity of system comparing with the traditional self-tuning controller. At the same time, because of fewer identification parameters, the computation is greatly simplified.

5.2. Industrial Heating Process Experiments. The experimental platform $U$ model smooth adaptive internal model controller was designed in an experimental platform for the heating process of a steel billet. The main electric equipment of the experimental filed includes a heating furnace, a piece of steel billet, an infrared temperature measuring mechanism, and SIMATIC ET200M and HMI (human machine interface). The experimental platform is shown in Figures 10 and 11.

The main parameters of the experimental settings are shown in Tables 2 and 3.

Like the simulation above, the initial parameters are configured according to Table 1 . The real temperature of steel billet and real electric heating system statements are shown from Figures 12 to 15 . Figures 12 and 13 are electric
TABLE 2: The indexes of duty cycle and heating power.

\begin{tabular}{lcc}
\hline Indexes & Duty cycle & Heating power \\
\hline 0 & $0: 1$ & $100 \%$ \\
1 & $1: 1$ & $50 \%$ \\
2 & $2: 1$ & $33.3 \%$ \\
3 & $3: 1$ & $25 \%$ \\
4 & $4: 1$ & $20 \%$ \\
999 & $999: 1$ & $0.1 \%$ \\
\hline
\end{tabular}

TABLE 3: The expected heating targets.

\begin{tabular}{lc}
\hline Initial parameters & Values \\
\hline Temperature target $\left({ }^{\circ} \mathrm{C}\right)$ & 400 \\
The length of the billet $(\mathrm{cm} * \mathrm{~cm} * \mathrm{~cm})$ & $20 * 10 * 5$ \\
Initial temperature of the billet $\left({ }^{\circ} \mathrm{C}\right)$ & 25 \\
Permissible temperature difference $\left({ }^{\circ} \mathrm{C}\right)$ & 20 \\
Preheating temperature of the electric heating & 120 \\
furnace $\left({ }^{\circ} \mathrm{C}\right)$ &
\end{tabular}

heating system with $U$ model smooth adaptive internal model controller and Figures 14 and 15 are electric heating system with traditional adaptive controller.

The industrial electric heating system is a typical industrial dynamic system with dead time and uncertain parameters. Compared to traditional adaptive controller, the $U$ model smooth adaptive internal model controller achieves more accuracy temperature control for its simplified identification structure.

\section{Conclusions}

This paper addresses the issue of modeling and control for a class of modern industrial control systems with timevarying model parameters. In order to improve the dynamic performance and control precision, the process calculation must be simplified. Therefore, the following conclusions are drawn:

(a) For the time-varying parameters, a smooth adaptive internal model control method based on $U$ model is proposed. Differing from traditional adaptive methods, the proposed controller can greatly simplify the identification process.

(b) Through the $U$ model parameter identification error equation, the stability requirements of the algorithm 


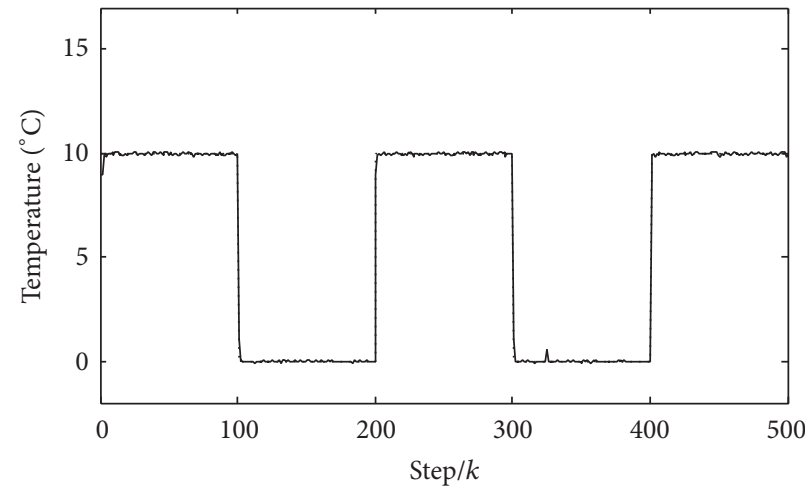

Reference trajectory

Smooth adaptive internal model

controller based on $U$ model

(a)

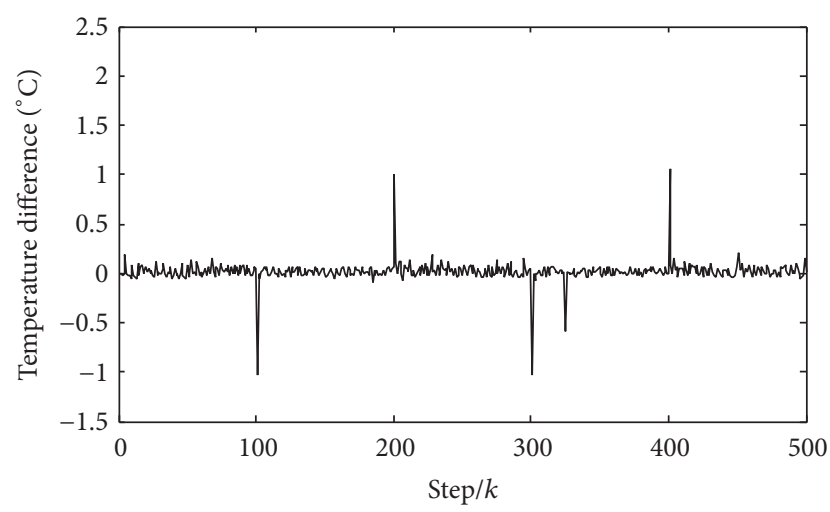

Smooth adaptive internal model controller based on $U$ model

(c)

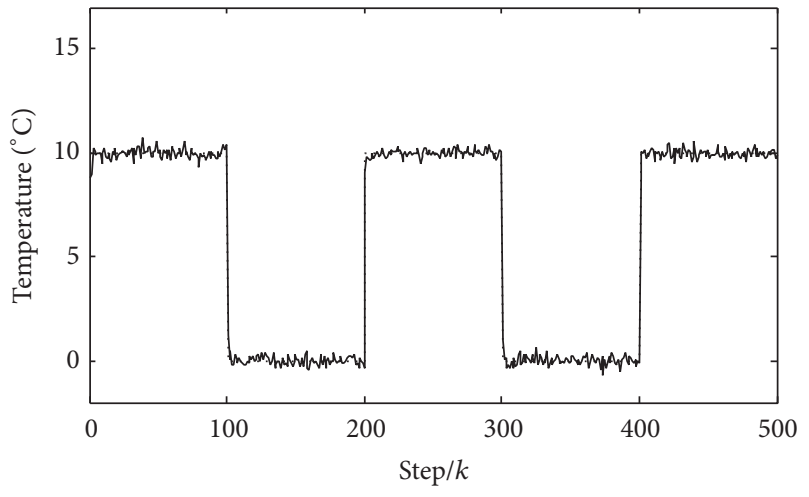

Reference trajectory

Traditional adaptive controller

(b)

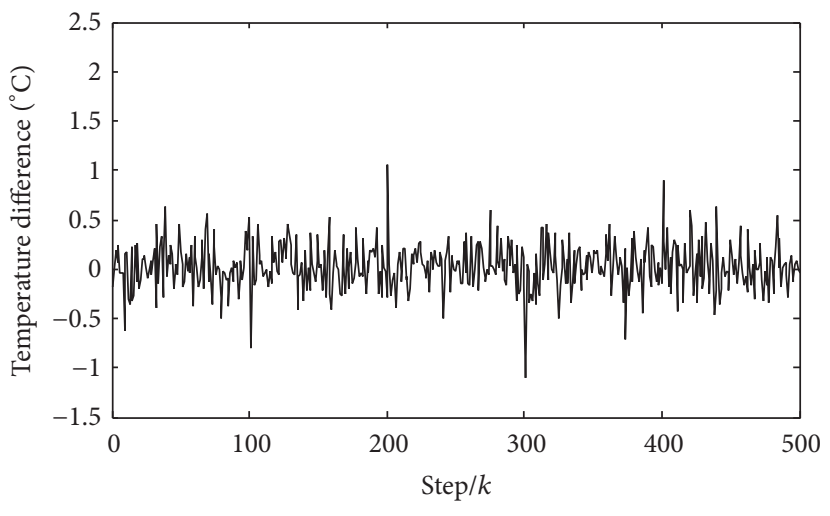

Traditional adaptive controller

FIgURE 9: Simulation results.

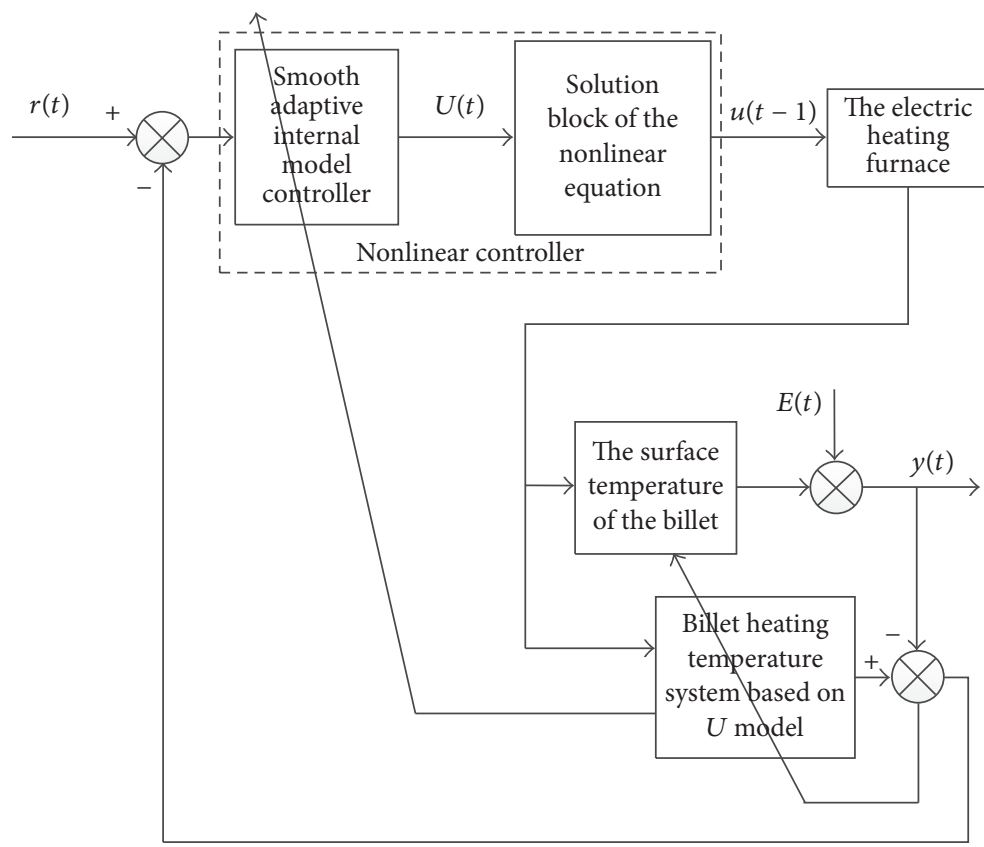

FIgURE 10: The control system of the experimental platform. 


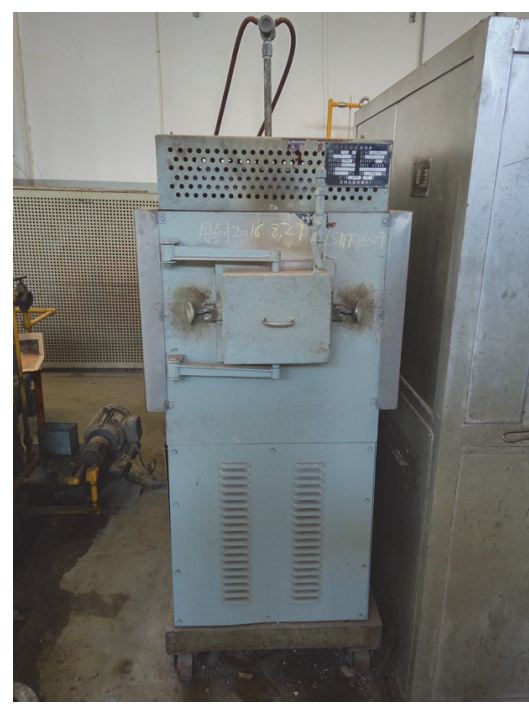

FIGURE 11: The experimental electric heating platform.

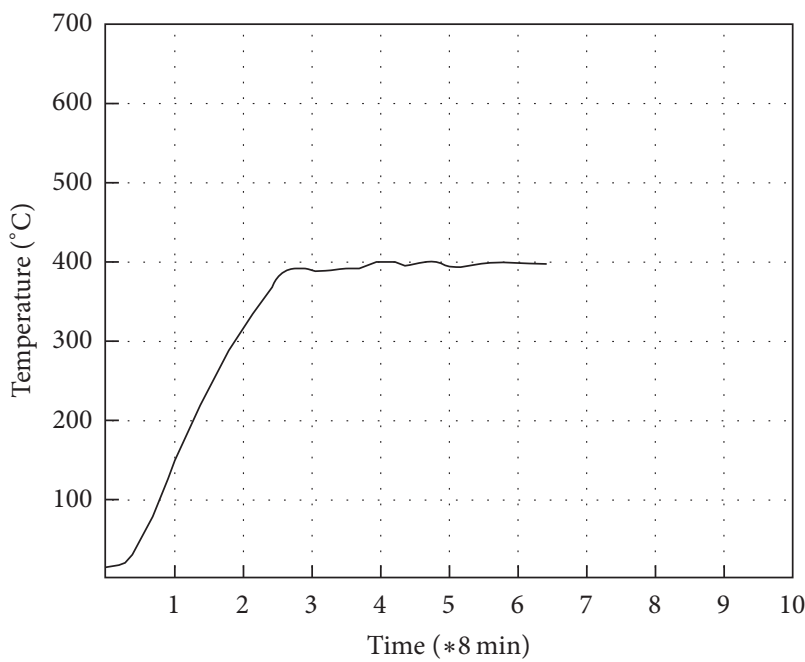

FIGURE 12: Real temperature of the steel billet with $U$ model smooth adaptive internal model controller.

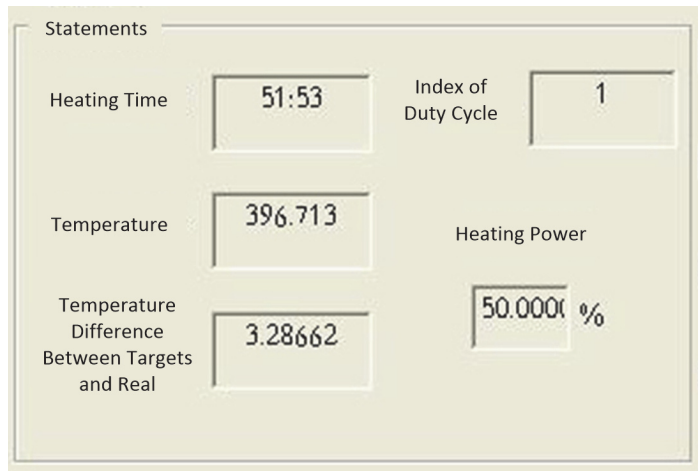

FIgURE 13: Real data of the experimental platform with $U$ model smooth adaptive internal model controller.

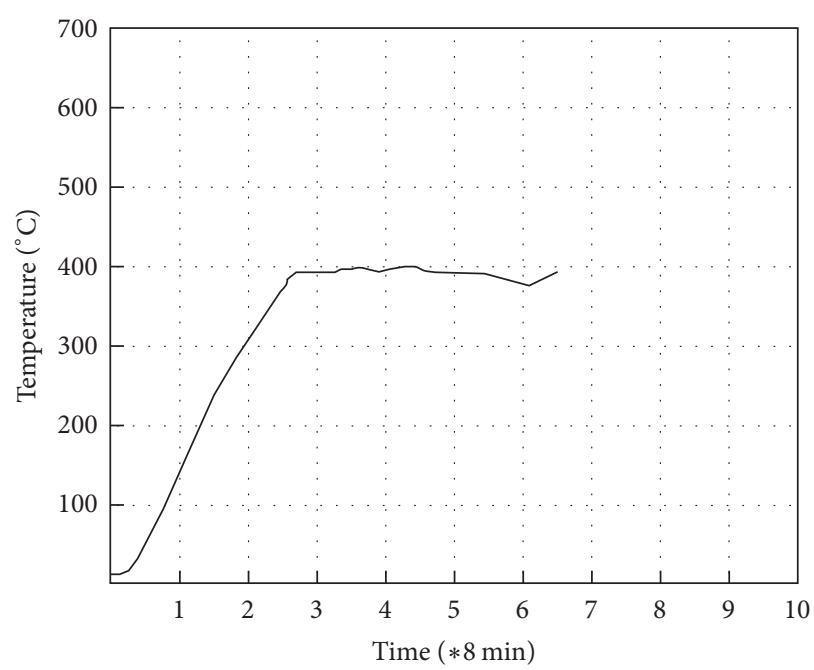

FIGURE 14: Real temperature of the steel billet with traditional adaptive controller.

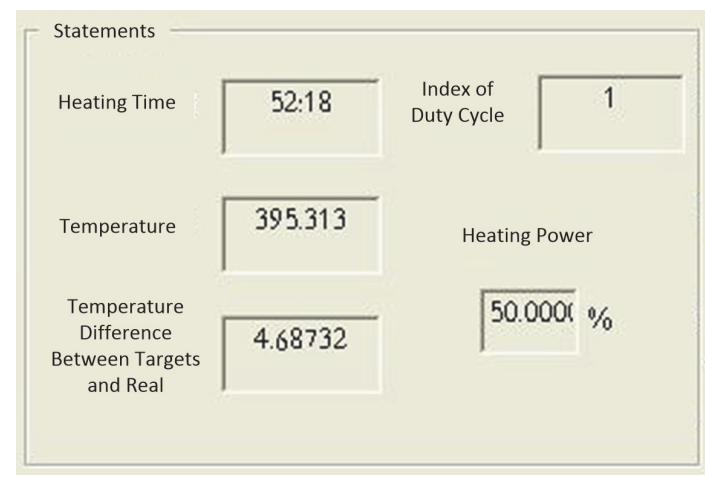

FIGURE 15: Real data of the experimental platform with traditional adaptive controller.

are achieved. With the small gain theorem and virtual equivalent system, the learning rate is analyzed and convergence proof of the closed-loop $U$ model system was achieved.

(c) The industrial control systems always suffer from unexpected fast disturbance. Simulation results of the nonlinear industrial heating process system shows that the proposed algorithm can greatly simplify the complexity of the identified systems, reduce the calculation, and realize the accuracy control for nonlinear systems.

(d) The practical experiment of heating system with the $U$ model smooth adaptive internal model controller verified the effectiveness of the proposed algorithm.

\section{Competing Interests}

The authors declare that they have no competing interests. 


\section{References}

[1] F. Xu, Y. Cheng, H. Ren, and S. Wang, "Research on adaptive neural network control system based on nonlinear U-model with time-varying delay," Mathematical Problems in Engineering, vol. 2014, Article ID 420713, 7 pages, 2014.

[2] K. Hassan, Nonlinear System, Pearson Education, New York, NY, USA, 3rd edition, 2002.

[3] Y. H. Tan and L. Deng, "Modeling the dynamic sandwich system with hysteresis using NARMAX model," Mathematics and Computers in Simulation, vol. 97, pp. 162-188, 2014.

[4] E.-W. Bai and D. Li, "Convergence of the iterative Hammerstein system identification algorithm," IEEE Transactions on Automatic Control, vol. 49, no. 11, pp. 1929-1940, 2004.

[5] B. S. Dayal and J. F. Macgregor, "Identification of finite impulse response models: methods and robustness issues," Industrial and Engineering Chemistry Research, vol. 35, no. 11, pp. 40784090, 1996.

[6] Q. S. Zheng and E. Zafiriou, "A local form of small gain theorem and analysis of feedback Volterra systems," IEEE Transactions on Automatic Control, vol. 44, no. 3, pp. 635-640, 1999.

[7] E.-W. Bai and K. Li, "Convergence of the iterative algorithm for a general Hammerstein system identification," Automatica, vol. 46, no. 11, pp. 1891-1896, 2010.

[8] D. W. Hu and Z. Z. Wang, "System identification of nonlinear wiener system," ACTA Automatica Sinica, no. 2, pp. 151-159, 1991.

[9] Q. M. Zhu and L. Z. Guo, "A pole placement controller for non-linear dynamic plants," Proceedings of the Institution of Mechanical Engineers. Part I: Journal of Systems and Control Engineering, vol. 216, no. 6, pp. 467-476, 2002.

[10] S. H. Jason, T. H. Tsai, and G. L. Long, "A modified NARMAX model-based self-tuner with fault tolerance for unknown nonlinear stochastic hybrid systems with an input-output direct feed-through term," ISA Transactions, vol. 53, no. 1, pp. 56-75, 2014.

[11] M. Shafiq and M. Haseebuddin, "U-model-based internal model control for non-linear dynamic plants," Proceedings of the Institution of Mechanical Engineers, Part I: Journal of Systems and Control Engineering, vol. 219, no. 6, pp. 449-458, 2005.

[12] S. S. Ali, M. A. Fouad, S. Muhammad et al., "MIMO U-model based control: real-time tracking control and feedback analysis via small gain theorem," WSEAS Transactions on Circuits and Systems, vol. 7, no. 7, pp. 610-619, 2008.

[13] F.-X. Xu, Q.-M. Zhu, D.-Y. Zhao, and S.-Y. Li, "U-model based design methods for nonlinear control systems a survey of the development in the 1st decade," Control and Decision, vol. 28, no. 7, pp. 961-977, 2013.

[14] Y. S. Liu, "Robust adaptive observer for nonlinear systems with unmodeled dynamics," Automatica, vol. 45, no. 8, pp. 1891-1895, 2009.

[15] W. Zhang, "Virtual equivalent system theory for self-tuning control," Journal of Harbin Institute of Technology, vol. 46, no. 5, pp. 107-112, 2014.

[16] H. X. Wu, "Application and development of adaptive control technology," Control Theory \& Applications, vol. 9, no. 2, pp. 110, 1992.

[17] Q. M. Zhu, D. Y. Zhao, and J. H. Zhang, "A general Ublock model-based design procedure for nonlinear polynomial control systems," International Journal of Systems Science, vol. 47, no. 14, pp. 3465-3475, 2016.
[18] W. Zhang, "On the stability and convergence of self-tuning control-virtual equivalent system approach," International Journal of Control, vol. 83, no. 5, pp. 879-896, 2010.

[19] X. Wu, L. Liu, Q. Zhu, W. Du, B. Wang, and J. Zhang, "Umodel-based adaptive control for a class of stochastic nonlinear dynamic plants with unknown parameters," International Journal of Modelling, Identification and Control, vol. 13, no. 3, pp. 135-143, 2011.

[20] Y. Y. He and M. D. Yan, Nonlinear Control System Theory and Applications, Xidian University Press, Xian, China, 2007. 


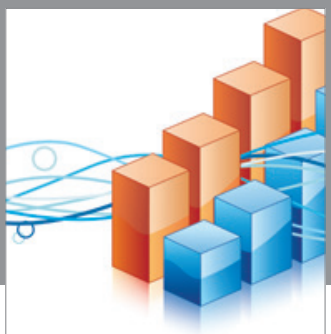

Advances in

Operations Research

vatem alat4

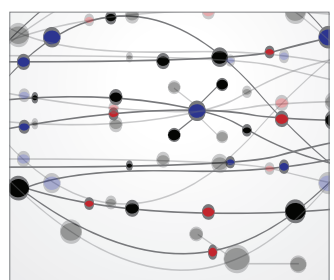

\section{The Scientific} World Journal
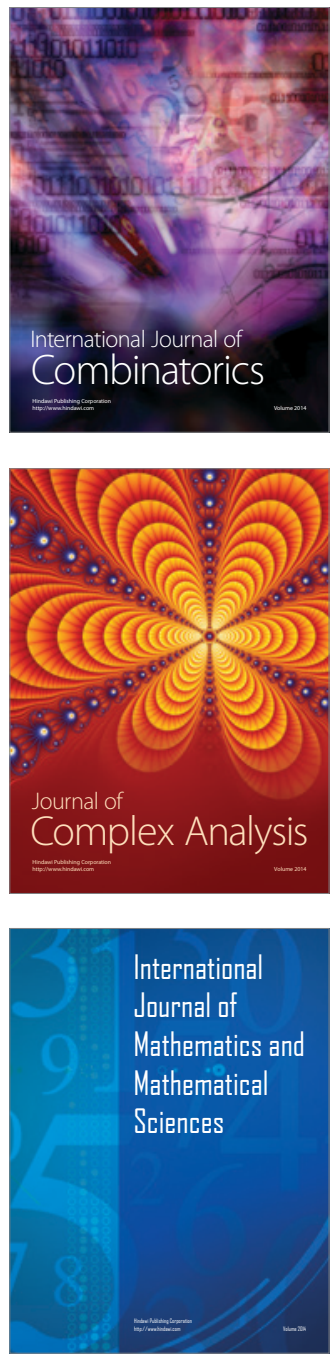
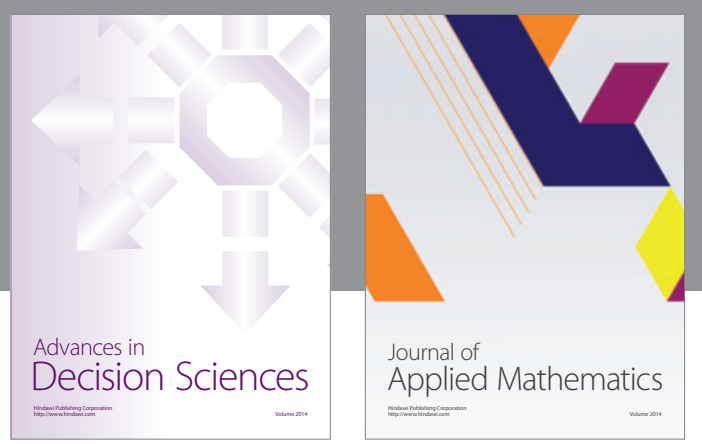

Algebra

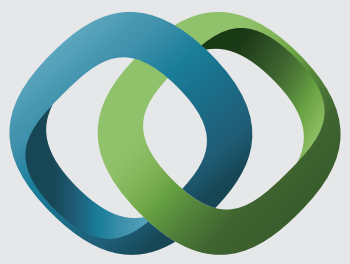

\section{Hindawi}

Submit your manuscripts at

http://www.hindawi.com
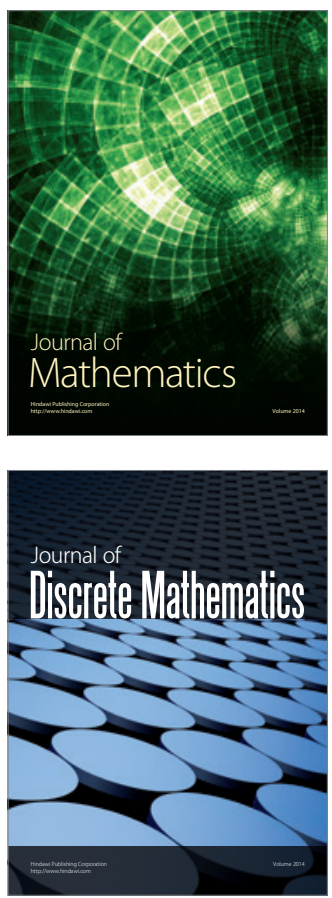

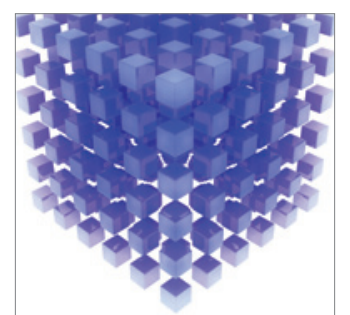

Mathematical Problems in Engineering
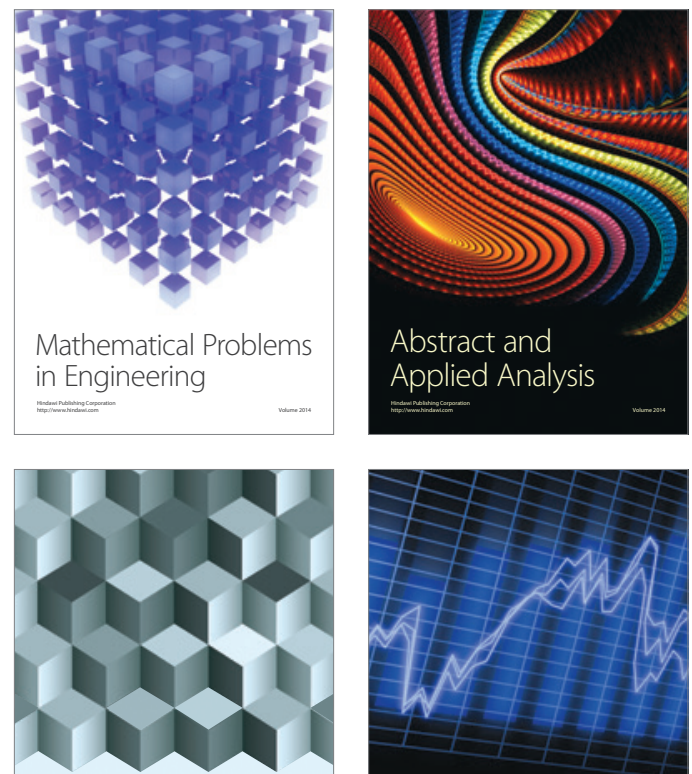

Journal of

Function Spaces

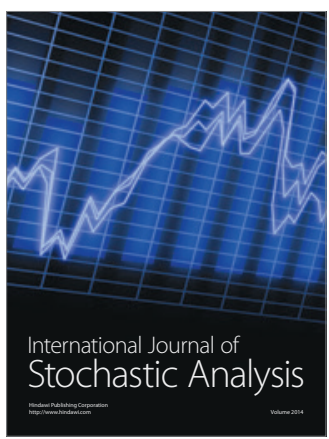

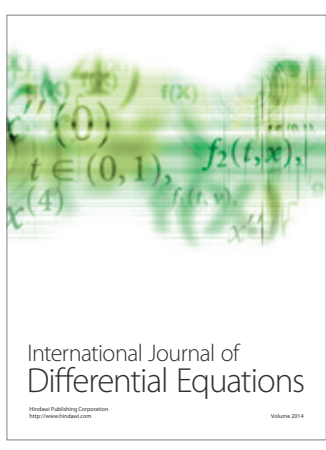
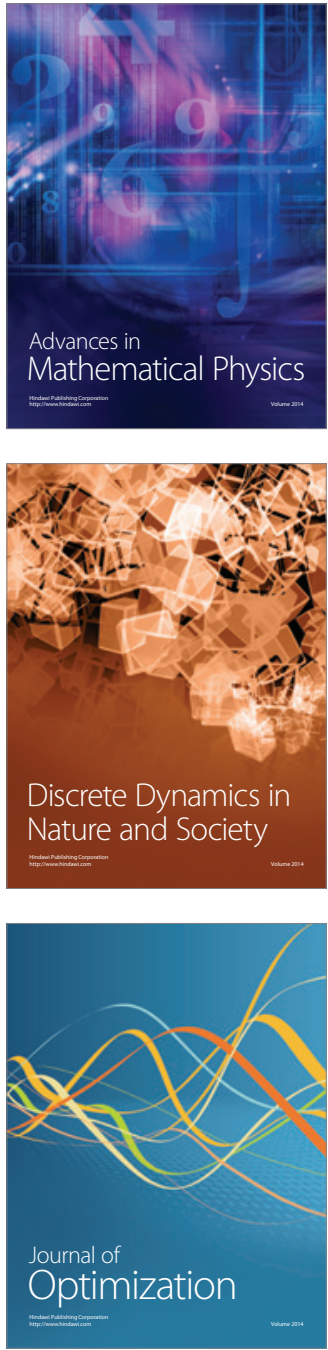